\title{
Hemianopic and Quadrantanopic Field Loss, Eye and Head Movements, and Driving
}

\author{
Joanne M. Wood, ${ }^{1,2}$ Gerald McGwin Jr, ${ }^{3}$ Jennifer Elgin, ${ }^{3}$ Michael S. Vaphiades, ${ }^{3,4}$ \\ Ronald A. Braswell, ${ }^{3}$ Dawn K. DeCarlo, ${ }^{3}$ Lanning B. Kline, ${ }^{3}$ and Cynthia Owsley ${ }^{3}$
}

\begin{abstract}
Purpose. To compare eye and head movements, lane keeping, and vehicle control of drivers with hemianopic and quadrantanopic field defects with controls, and to identify differences in these parameters between hemianopic and quadrantanopic drivers rated safe to drive by a clinical driving rehabilitation specialist compared with those rated as unsafe.
\end{abstract}

Methods. Eye and head movements and lane keeping were rated in 22 persons with homonymous hemianopic defects and 8 with quadrantanopic defects (mean age, 53 years) who were $\geq 6$ months post-injury and 30 persons with normal fields (mean age, 53 years). All were licensed to drive and were current drivers or aimed to resume driving. Participants drove a 6.3-mile route along non-interstate city roads under in-traffic conditions. Vehicle control was assessed objectively by vehicle instrumentation for speed, braking, acceleration, and cornering.

Results. As a group, drivers with hemianopic or quadrantanopic defects drove slower, exhibited less excessive cornering or acceleration, and executed more shoulder movements than the controls. Those drivers with hemianopic or quadrantanopic defects rated as safe also made more head movements into their blind field, received superior ratings regarding eye movement extent and lane position stability, and exhibited less sudden braking and drove faster than those rated unsafe.

Conclusions. Persons with hemianopic and quadrantanopic defects rated as safe to drive compensated by making more head movements into their blind field, combined with more stable lane keeping and less sudden braking. Future research should evaluate whether these characteristics could be trained in rehabilitation programs aimed at improving driving safety in this population. (Invest Ophthalmol Vis Sci. 2011;52:1220-1225) DOI:10.1167/ iovs.10-6296

$\mathrm{T}$ here has been considerable debate in the literature regarding the driving safety of individuals with homonymous hemianopic and quadrantanopic field defects. ${ }^{1-5}$ An important consideration for understanding their driving performance is

From the ${ }^{1}$ School of Optometry and ${ }^{2}$ Institute of Health and Biomedical Innovation, Queensland University of Technology, Brisbane, Australia; and Departments of ${ }^{3}$ Ophthalmology and ${ }^{4}$ Neurology, School of Medicine, University of Alabama at Birmingham, Birmingham, Alabama.

Supported by National Institutes of Health Grants R21-EY14071 and P30-AG22838, EyeSight Foundation of Alabama, Research to Prevent Blindness Inc., and a QUT Professional Development Leave grant.

Submitted for publication July 29, 2010; revised October 13, 2010; accepted November 9, 2010.

Disclosure: J.M. Wood, None; G. McGwin Jr, None; J. Elgin, None; M.S. Vaphiades, None; R.A. Braswell, None; D.K. DeCarlo, None; L.B. Kline, None; C. Owsley, None

Corresponding author: Joanne M. Wood, Victoria Park Rd., Kelvin Grove, Brisbane, Queensland, Australia Q4059; j.wood@qut.edu.au. the extent to which individuals with these field defects might adopt patterns of eye and head movements that assist them to compensate for their field loss. If this were the case, it would provide justification for exploring the potential for predicting whether an individual with these field defects might have the potential for safe driving and for training scanning behaviors as a means of improving driver safety in these individuals. ${ }^{6-10}$

Numerous studies have explored the eye and head movements and scanning behavior of persons with homonymous hemianopia in well-controlled laboratory-based settings; however, none have been conducted under real-world driving conditions. These laboratory-based studies have shown that persons with hemianopic field defects mainly look toward their blind hemifield when undertaking a range of tasks, including counting dots, ${ }^{11-13}$ viewing natural and degraded images, ${ }^{14}$ viewing randomly presented ${ }^{13}$ and moving targets ${ }^{15}$ within a virtual reality environment, but not when assembling wooden models in a static environment. ${ }^{11}$ Martin et al. ${ }^{11}$ explain their findings by suggesting that these compensatory strategies of biasing gaze in the direction of the blind hemifield are most evident in dynamic and unpredictable environments, where subjects cannot rely on their spatial memory to locate salient objects. This hypothesis was recently supported by Hardiess et al., ${ }^{13}$ who found that the differences in gaze patterns between hemianopes who performed visual search tasks at "adequate" or "inadequate" levels were dependent on the level of complexity of the search task. They suggested that the poorer performance of the inadequate performers on the more complex task was due to reduced working memory. Given that driving is a complex and dynamic task, where drivers cannot rely on their spatial memory to locate salient objects, we hypothesize that individuals with hemianopic defects might similarly adopt head and eye movements that bias fixation toward the blind field while driving, and that those who adopt these strategies will be able to successfully compensate for their field defects and exhibit safer driving performance.

In addition, few studies have assessed the on-road driving characteristics of hemianopic and quadrantanopic drivers, including speed, braking, acceleration, cornering, and lane keeping, which might also differentiate between safe and unsafe drivers. Szlyk et al. ${ }^{1}$ in an interactive driving simulator study reported higher numbers of lane boundary crossings for a small sample of persons with hemianopia compared with controls, while on-road studies have also reported problems with steering stability and lane keeping. ${ }^{2,5}$ Bowers et al. ${ }^{16}$ in a driving simulator study also showed that hemianopic persons adopted a lane position toward their seeing field, therefore providing a safety margin on their blind side. However, this finding has not been verified for actual on-road driving performance, an issue that is addressed in this study.

The aim of the present study was thus to compare the patterns of eye and head movements, lane keeping, and vehicle control of drivers with homonymous hemianopia and quadrantanopia to that of age-matched drivers with normal visual fields 
while driving under real-world conditions. We also compared the eye and head movements of those hemianopic and quadrantanic drivers rated as safe to drive with those rated as unsafe. We hypothesized, based on the evidence of previous studies, that persons with hemianopia would make more head movements into their blind field as a means of compensating for their field defects and that this would be more evident in those rated as safe to drive. We also hypothesized that those rated as unsafe to drive would adopt a lane position in the direction of their seeing field to avoid their blind side, while those rated as safe to drive would maintain a relatively central lane position.

\section{METHODS}

\section{Participants}

Participants included 22 persons with homonymous hemianopia, eight persons with homonymous quadrantanopic visual field defects (mean age, $52.7 \pm 19.8$ years), and 30 age-matched control participants (mean age, $52.5 \pm 19.1$ years). All participants were current drivers or had driven in the last two years before enrollment in the study and were legally licensed to drive and had visual acuity of $20 / 60$ or better in at least one eye (vision requirement for licensure in Alabama). Exclusion criteria were Parkinson's disease, multiple sclerosis, Alzheimer's disease, hemiparesis, ocular or neurologic conditions resulting in visual field defects (other than hemianopia or quadrantanopia in the field defect group), and lateral spatial neglect as defined by the Star Cancellation Test. ${ }^{17}$

A detailed description of the visual field characteristics and etiology of brain injury for the participants with hemianopic and quadrantanopic field defects is presented elsewhere. ${ }^{5}$ In summary, for the participants with hemianopic field defects, there were five with right hemianopic loss and 17 with left hemianopic loss, and eight of the 22 had macular sparing. For the participants with quadrantanopia, half had left-sided loss and half right-sided loss, with five with superior loss and three with inferior field loss. The most common underlying etiology of field loss was cerebrovascular accident (60\%), with the remaining causes being trauma, tumors, arteriovenous malformation, and congenital abnormalities.

The protocol was approved by the Institutional Review Board for Human Use at the University of Alabama at Birmingham and adhered to the tenets of the Declaration of Helsinki. After the purpose of the study was explained, participants were asked to sign a document of informed consent before enrolling.

\section{Instrumented Measures of Driving}

On-road driving performance was assessed under in-traffic conditions in an automatic transmission vehicle (Chevrolet Impala 2007), instrumented to measure acceleration and deceleration, lateral/ longitudinal forces, vehicle speed, and recording of the internal and external driving environment (Vigil Vanguard System, Brisbane, Australia). An accelerometer was mounted on the roof of the vehicle along with inertial sensors to record braking and acceleration forces, while a roof-mounted GPS system sampled the speed and position of the vehicle. Three cameras were mounted on the roof of the vehicle (one each to the extreme left and right of the vehicle and pointing slightly downwards to record the position of the vehicle front right and left fenders for assessment of lane position), and one mounted in the center of the vehicle to record the forward road scene.

An internally mounted camera pointing directly toward the participant's face and upper torso was used to record the pattern of head and eye movements from which an index of eye and head movements was derived post-testing. Although this does not provide a quantitative analysis of fixation durations, saccades, and head movements, it provides a good basis for identifying and further exploring any differences in eye and head movement patterns between drivers with hemianopic or quadrantanopic field defects and those with normal fields. This was necessary because recording eye movements in the field, under ever-changing outdoors conditions while the participant is actually driving, is much more challenging than in a laboratory setting or driving simulators where there is excellent level of control of lighting and participant location relative to the scene ahead.

The driving performance of each participant was assessed under in-traffic conditions along 6.3 miles of non-interstate driving in residential and commercial areas of a city as described previously. ${ }^{5,18}$ Drives were held between $9 \mathrm{AM}$ and 3 PM to avoid rush hour traffic and were cancelled if it was raining or the road was wet. A certified driving rehabilitation specialist (CDRS) who was also a licensed occupational therapist sat in the front passenger seat of the vehicle; she has eight years of clinical experience in driving assessment and rehabilitation of patients with a wide variety of medical and neurologic conditions. The CDRS evaluated driving performance, had access to a dual brake, and was responsible for monitoring safety and was aware of the medical and functional characteristics of the participants she was evaluating on the road, as is standard practice. However, because of the potential for bias and its impact on interpreting the results, we were also interested in the extent to which her ratings of driving safety agreed with two backseat raters who ensured appropriate operation of the vehicle's instrumentation and recording system throughout the drive and were completely masked to the visual field (i.e., hemianopia/ quadrantanopia/normal) and health characteristics of each participant.

Each drive began by participants completing a series of basic driving maneuvers in a parking lot to ensure they had adequate vehicle control and to become familiar with the vehicle. Once the participant exhibited adequate vehicle control, the on-road driving evaluation began, starting in quiet city streets in a residential neighborhood and then proceeding to busier roads. The CDRS used a five-point rating system to assess different components of driving performance, as well as to derive an overall rating of performance: $1=$ driving was so unsafe that the drive was terminated; $2=$ exhibited a couple of unsafe maneuvers but did not reach the level of drive termination; 3 = driving was unsatisfactory but not unsafe at that time given the traffic circumstances; $4=$ driver exhibited a few minor driving errors; and $5=$ there were no obvious driving errors. ${ }^{18}$ Scores of 1 and 2 were classified as failing the driving assessment and being unsafe to drive, whereas scores of 3, 4, and 5 were considered to be passes.

There was perfect agreement between the CDRS and the backseat evaluator in terms of determining which of the drivers passed or failed the driving assessment, ${ }^{18}$ which provides important validation regarding the reliability of the CDRS's judgments with respect to safe driving (the study's main dependent variable).

\section{Post-test Scoring of Video Footage}

The data collected by the instrumented vehicle were exported as text and graphical files and examined using specialized software (Vigil Vanguard System), which automatically generated outcome scores of driving speed and excessive force events defined as jerky cornering, sudden braking, and acceleration. Excessive or jerky acceleration, braking, and cornering were defined as when $>0.2 g$ was exerted and recorded by the detecting sensors. This value was set as the default by the system, being defined as the force level that typically feels uncomfortable to a passenger riding in a vehicle.

The videos of the external environment were analyzed to rate road position and those of the internal vehicle environment to count head movements and rate eye movements. Two independent research assistants who were completely masked to the visual field (i.e., hemianopia/quadrantanopia/normal), and health characteristics of each participant, or their driving category (safe/unsafe), conducted an analysis of the driving videos using a scoring system that allowed quantitative 
scoring of head movements, categorization of the extent of eye movements, and rating of lane positioning (given that the lane markings along the route were clearly evident only for some sections of the driving route). Sideways head movements were categorized into small and large head movements, where small head movements were defined as movements ranging from the forward-facing position to a $45^{\circ}$ angle (selected as the halfway position between a forward facing position and one where the driver was looking directly sideways at $90^{\circ}$ to the camera view), with large head movements defined as those that were greater than $45^{\circ}$. Head movements that were around the borderline $45^{\circ}$ position, where it was difficult to determine whether they fell into the small or large categories, were always classified as small for the purposes of consistency. Counts were then made of each movement by category and direction (left or right). Vertical head movements were counted, as were shoulder movements.

A five-point Likert-type scale was used to categorize eye movements $(1=$ few saccades, $3=$ average number of saccades, $5=$ many saccades), road position $(1=$ very poor/unstable, $3=$ some errors, $5=$ very good/stable and whether a central position or to the left or the right of the lane for the majority of the drive), and head movements overall ( 1 = not excursive, $3=$ some excursions, $5=$ highly excursive). These categorizations were made once the raters had observed all the videos and so had a clear impression of the range of performance across all participants.

The intra-rater reliability of the two research assistants scoring the driving videos ranged from $r=0.51, P<0.0001$, for small right head movements to $r=0.85 ; P<0.0001$, for the total number of head movements. The data from the two raters were thus combined to provide an average rating for all the head movement counts and overall scoring of head and eye movements and lane position. Driving videos from a random sample of nine participants were reanalyzed by the research assistants to derive a measure of their own scoring repeatability that ranged from intraclass correlation coefficient (ICC) $=0.65, P<$ 0.0001 , to $\mathrm{ICC}=0.97, P<0.0001$

\section{Analysis}

Analysis of Variance and Kruskal-Wallis tests were used to compare driving performance between participant groups. $P \leq 0.05$ (two-sided) was considered statistically significant.

\section{Results}

The data for the number and direction of head movements and the ratings of eye and head movements and lane positioning are given in Table 1 as a function of field loss and whether the drivers were rated as safe or unsafe to drive by the CDRS ( 23 of the participants with field defects were rated as safe to drive and seven as unsafe ${ }^{5}$ ). When considered as a group, the drivers with hemianopic field defects tended to exhibit larger numbers of head movements than either those with quadrantanopic field defects or normal visual fields, particularly for large head movements; however, these differences failed to reach statistical significance. The hemianopic participants made significantly more shoulder movements than did the controls; however, no other differences were significant. There were no between-group differences in any of the ratings of eye and head movements or lane position.

When the data for the participants with field defects were considered based on whether they were rated as safe or unsafe to drive by the CDRS (Table 1), both the ratings of eye movements and lane position were significantly higher (better performance) for the safe than the unsafe drivers, where the safe drivers were rated as exhibiting significantly more excursive eye movements and maintaining a more stable lane position than those rated as unsafe. There was also a trend for those participants rated as safe to drive to exhibit more head movements than those rated as unsafe, although these differences failed to reach significance.

When the head movement data were analyzed as a function of whether the field defects were right or left sided for the hemianopic participants alone, and then grouped with the quadrantanopic participants who had left- or right-sided loss (Table 2), there were generally more head movements in the direction of the blind hemifield, particularly for those with a left-sided defect. These differences reached marginal significance $(P=0.0519)$ for small leftward head movements for those with left-sided hemianopic loss (where those with left hemianopic loss made more than twice the number of head movements into the left field than did the right hemianopes). Whether this bias to the left was exacerbated by the need to look in the direction of oncoming traffic when driving on the right side in the interests of safety was explored by analyzing the number of left- and right-sided head movements as a function of whether participants were rated as safe or unsafe to drive, including both the cases and the control participants (Table 2). These data show there was a nonsignificant leftward bias for small head movements, where more head movements were made into the left field regardless of field loss and sug-

TABle 1. Group Mean Data for the Head Movement Counts and Overall Ratings of Eye and Head Movements and Lane Position for Those with Hemianopic, Quadrantanopic Field Defects and Normal Fields, and Then for the Field Defect Group Based on Whether They Were Rated As Safe or Unsafe to Drive

Field Loss Patients

\begin{tabular}{|c|c|c|c|c|c|c|c|}
\hline Counts & $\begin{array}{l}\text { Hemianopes } \\
\quad(n=22)\end{array}$ & $\begin{array}{l}\text { Quadrantanopes } \\
\qquad(n=8)\end{array}$ & $\begin{array}{l}\text { Normal Fields } \\
\quad(n=30)\end{array}$ & $\boldsymbol{P}$ & $\begin{array}{c}\text { Safe } \\
(n=23)\end{array}$ & $\begin{array}{l}\text { Unsafe } \\
(n=7)\end{array}$ & $\boldsymbol{P}$ \\
\hline Head movements (large) left & 14.45 & 11.06 & 11.18 & 0.0632 & 13.95 & 12.21 & 0.5196 \\
\hline Head movements (large) right & 11.02 & 9.63 & 9.02 & 0.0914 & 10.87 & 9.93 & 0.5438 \\
\hline Head movements (small) left & 20.43 & 18.63 & 19.73 & 0.9126 & 21.46 & 15.00 & 0.2147 \\
\hline Head movements (small) right & 11.73 & 11.63 & 10.32 & 0.3953 & 11.98 & 10.79 & 0.4546 \\
\hline Total head movements & 57.81 & 51.06 & 50.17 & 0.2522 & 58.26 & 47.93 & 0.2184 \\
\hline Shoulder movements & 5.45 & 4.00 & 3.37 & $0.0110^{*}$ & 4.97 & 5.36 & 0.7809 \\
\hline Vertical head movements & 2.27 & 2.50 & 2.42 & 0.9622 & 2.46 & 1.93 & 0.5158 \\
\hline \multicolumn{8}{|l|}{ Summary ratings $\dagger$} \\
\hline Rating eye movements & 3.00 & 2.79 & 3.04 & $0.4234 \ddagger$ & 3.10 & 2.50 & $0.0145^{*} \ddagger$ \\
\hline Rating road position & 3.05 & 3.19 & 3.33 & $0.5373 \ddagger$ & 3.33 & 2.29 & $0.0108^{*} \ddagger$ \\
\hline Rating head movements & 3.27 & 2.87 & 2.90 & $0.3231 \ddagger$ & 3.24 & 2.93 & $0.4247 \ddagger$ \\
\hline
\end{tabular}

$* P<0.05$.

$\dagger$ Based on a five-point rating scale (1-5), where 5 represents better performance.

$\ddagger$ Kruskal-Wallis test. 
TABle 2. Group Mean Data for Head Movement Counts for the Hemianopic Participants and the Hemianopic and Quadrantanopic Participants Combined Dependent on Whether Their Field Defect Was on the Right or Left Side

\begin{tabular}{|c|c|c|c|c|c|c|c|c|c|}
\hline \multirow[b]{3}{*}{ Counts } & \multirow{2}{*}{\multicolumn{2}{|c|}{$\frac{\text { Side of Field Defect }}{\text { (Hemianopes Only) }}$}} & \multirow[b]{3}{*}{$\boldsymbol{P}$} & \multirow{2}{*}{\multicolumn{2}{|c|}{$\begin{array}{c}\text { Side of Field Defect } \\
\begin{array}{c}\text { (Hemianopes and } \\
\text { Quadrantanopes } \\
\text { Combined) }\end{array}\end{array}$}} & \multirow[b]{3}{*}{$\boldsymbol{P}$} & & & \multirow[b]{3}{*}{$\boldsymbol{P}$} \\
\hline & & & & & & & \multicolumn{2}{|c|}{ All Participants } & \\
\hline & $\begin{array}{c}\text { Left } \\
(n=17)\end{array}$ & $\begin{array}{c}\text { Right } \\
(n=5)\end{array}$ & & $\begin{array}{c}\text { Left } \\
(n=21)\end{array}$ & $\begin{array}{c}\text { Right } \\
(n=9)\end{array}$ & & $\begin{array}{c}\text { Safe } \\
(n=53)\end{array}$ & $\begin{array}{c}\text { Unsafe } \\
(n=7)\end{array}$ & \\
\hline Head movements (large) left & 15.03 & 12.50 & 0.4597 & 14.38 & 11.61 & 0.3605 & 12.39 & 12.21 & 0.8904 \\
\hline Head movements (large) right & 10.38 & 13.20 & 0.1344 & 10.07 & 12.00 & 0.2142 & 9.82 & 9.93 & 0.9450 \\
\hline Head movements (small) left & 23.24 & 10.90 & 0.0519 & 22.64 & 13.67 & 0.0877 & 20.48 & 15.00 & 0.0654 \\
\hline Head movements (small) right & 11.68 & 11.90 & 0.9008 & 11.47 & 12.22 & 0.5299 & 11.04 & 10.79 & 0.9634 \\
\hline Total head movements & 60.56 & 48.50 & 0.2617 & 58.76 & 49.61 & 0.5022 & 53.77 & 47.93 & 0.2885 \\
\hline
\end{tabular}

The data are also presented for all participants as a function of whether they were rated as safe or unsafe to drive.

gests that the bias toward the blind field of left-sided hemianopes and the lack of bias in those with right hemianopia may potentially be mediated by the need to fixate in the direction of oncoming traffic into the left field.

The data were then examined to specifically explore whether more head movements were indeed made into the blind than the seeing fields. Table 3 shows that as a group the hemianopic and quadrantanopic participants made significantly more head movements into their blind than seeing field. On average, $59 \%$ of all head movements were made into the blind compared with the seeing field ( 33.18 vs. 22.66), with $30 \%$ more large head movements made into the blind compared with the seeing field (13.67 vs. 10.53$)$ and $60 \%$ more small head movements made into the blind relative to the seeing field (19.51 vs. 12.13). When the hemianopic and quadrantanopic participants were considered based on whether they were rated as safe and unsafe to drive, only those drivers rated safe to drive made significantly more head movements on average into the blind than seeing field, whereas the differences were not significant for the unsafe drivers. These differences were most apparent for small head movements, where the safe drivers made $70 \%$ more small head movements into their blind than seeing field (21.13 vs. 12.30 ), whereas the unsafe drivers made only $20 \%$ more small head movements into their blind than seeing field (14.21 vs 11.57). In addition to this difference in distribution of head movements, those drivers rated as safe to drive made $50 \%$ more small head movements into their blind field relative to their seeing field compared with the unsafe drivers (21.13 vs. 14.21).
The lane-keeping data for the drivers with field defects were also scored according to whether the driver maintained a central position in the lane throughout the drive or drove either toward the right- or the left-hand side of the lane. For the seven hemianopic drivers rated as unsafe, six were rated as clearly driving toward the left or the right of the lane. In all cases, this was toward the seeing field, where the three persons with right hemianopia drove toward the left side of the lane and the three persons with left hemianopia drove toward the right side of the lane. Only one of the unsafe drivers maintained a central lane position (but their drive was terminated early because of safety concerns). For the 23 safe drivers, 19 maintained a central driving position, while four drove into the direction of their seeing field.

The reports generated by the instrumentation in the vehicle are summarized in Table 4 and demonstrate that the drivers with field defects drove significantly more slowly than did those with normal fields. The drivers with field loss drove significantly more in the $0-50 \mathrm{~km} /$ hour speed band (almost $90 \%$ of the drive) compared with $82 \%$ of the controls, and drove significantly less in the 50-70 km/hour speed band (10\% of the drive compared with $17 \%$ for the controls). We therefore looked at the data for the $0-50$ $\mathrm{km} /$ hour speed band, given that $90 \%$ of this group drove in this range. Interestingly, as well as driving more slowly than the controls, the participants with hemianopic and quadrantanopic field defects exhibited significantly less jerky acceleration and cornering than did those with normal fields. However, although the unsafe hemianopic and quadrantan-

TABlE 3. Group Mean Data for Head Movement Counts for the Hemianopic and Quadrantanopic Participants Combined Dependent on Whether Their Field Defect Was on the Blind or Seeing Side

\begin{tabular}{|c|c|c|c|c|c|c|c|c|c|}
\hline \multirow[b]{3}{*}{ Counts } & \multirow{2}{*}{\multicolumn{2}{|c|}{$\begin{array}{c}\begin{array}{c}\text { Head Movements } \\
\text { (Large) }\end{array} \\
\begin{array}{c}\text { Side of Field } \\
\text { Defect }\end{array}\end{array}$}} & \multirow[b]{3}{*}{$\boldsymbol{P}$} & \multirow{2}{*}{\multicolumn{2}{|c|}{$\begin{array}{c}\begin{array}{c}\text { Head Movements } \\
\text { (Small) }\end{array} \\
\begin{array}{c}\text { Side of Field } \\
\text { Defect }\end{array}\end{array}$}} & \multirow[b]{3}{*}{$\boldsymbol{P}$} & \multirow{2}{*}{\multicolumn{2}{|c|}{$\begin{array}{c}\text { All Head } \\
\text { Movements } \\
\begin{array}{c}\text { Side of Field } \\
\text { Defect }\end{array}\end{array}$}} & \multirow[b]{3}{*}{$\boldsymbol{P}$} \\
\hline & & & & & & & & & \\
\hline & Blind & Seeing & & Blind & Seeing & & Blind & Seeing & \\
\hline \multicolumn{10}{|l|}{ All hemianopes and } \\
\hline Safe $(n=23)$ & 14.06 & 10.76 & $0.0097^{*}$ & 21.13 & 12.30 & $0.0005^{*}$ & 35.20 & 23.07 & $0.0006^{*}$ \\
\hline Unsafe $(n=7)$ & 12.36 & 9.78 & 0.1770 & 14.21 & 11.57 & 0.5932 & 26.57 & 21.36 & 0.4173 \\
\hline
\end{tabular}

The data are also presented for the hemianopic and quadrantanopic participants as a function of whether they were rated as safe or unsafe to drive.

$$
{ }^{*} P<0.01 \text {. }
$$


TABle 4. Group Mean Data for the Outcome Measures for the Automated Scores Derived from the Vigil Vanguard System for the Field Defect Groups Compared to Those with Normal Fields

\begin{tabular}{|c|c|c|c|c|c|c|c|}
\hline & \multirow[b]{2}{*}{$\begin{array}{l}\text { Hemianopes } \\
\quad(n=22)\end{array}$} & \multirow[b]{2}{*}{$\begin{array}{l}\text { Quadrantanopes } \\
\qquad(n=8)\end{array}$} & \multirow[b]{2}{*}{$\begin{array}{l}\text { Normal Fields } \\
\quad(n=30)\end{array}$} & \multirow[b]{2}{*}{$\boldsymbol{P}$} & \multicolumn{2}{|c|}{ Field Loss Patients } & \multirow[b]{2}{*}{$\boldsymbol{P}$} \\
\hline & & & & & $\begin{array}{c}\text { Safe } \\
(n=23)\end{array}$ & $\begin{array}{l}\text { Unsafe } \\
(n=7)\end{array}$ & \\
\hline$\%$ course spent $0-50 \mathrm{~km} / \mathrm{hr}$ & 89.86 & 86.88 & 82.43 & $<0.0001^{*}$ & 87.35 & 94.71 & $0.0006^{*}$ \\
\hline$\%$ course spent $50-70 \mathrm{~km} / \mathrm{hr}$ & 9.95 & 12.88 & 17.37 & $<0.0001^{*}$ & 12.39 & 5.29 & $0.0005^{*}$ \\
\hline Jerky acceleration $0-50 \mathrm{~km} / \mathrm{hr}$ & 2.22 & 2.88 & 3.83 & 0.0377 & 2.04 & 3.57 & 0.0805 \\
\hline Sudden braking $0-50 \mathrm{~km} / \mathrm{hr}$ & 2.00 & 2.50 & 2.83 & 0.4161 & 1.61 & 3.86 & $0.0066^{*}$ \\
\hline Jerky cornering 0-50 km/hr & 2.86 & 3.38 & 4.67 & 0.0108 & 3.09 & 2.71 & 0.5819 \\
\hline
\end{tabular}

${ }^{*} P<0.01$.

opic drivers drove more slowly than did those who were rated as safe to drive, they exhibited twice as many sudden braking events than did the safe drivers, a difference that reached significance.

\section{Discussion}

In this study we compared the patterns of eye and head movements, lane keeping, and vehicle control of drivers with homonymous hemianopic and quadrantanopic field defects with that of age-matched control participants with normal fields while they were driving under real-world conditions. The hemianopic drivers made significantly more shoulder movements than did those with normal visual fields, and there was a trend for more head movements than either those with quadrantanopia or normal visual fields; however, these differences failed to reach significance, possibly because of the relatively small sample size. Those hemianopic and quadrantanopic drivers who were rated as safe to drive made significantly more head movements into their blind than seeing field and received superior ratings in the extent of their eye movements and stability of lane position than those rated as unsafe; unsafe drivers also drove toward their seeing field rather than maintaining a central lane position.

Our finding of more excursive eye movements for patients with field loss who were rated as safe to drive is in general accord with previous laboratory studies that have demonstrated that persons with homonymous hemianopia undertake increased numbers of fixations, longer search times, and longer fixation durations than controls when completing visual search tasks. ${ }^{7}$ Interestingly, in our study we found greater numbers of head movements were made in the direction of the blind hemifield for the hemianopic and qaudranatanopic drivers when considered as a group and particularly for those rated as safe to drive. These findings support our initial hypothesis based on previous laboratory-based studies reporting that persons with hemianopia spend most of their time looking toward their blind hemifield when undertaking a range of tasks. ${ }^{11,12,14,15}$ These compensatory strategies of biasing gaze in the direction of the blind hemifield have also been shown to be most evident when hemianopic subjects are in dynamic and unpredictable environments, where the subjects cannot rely on their spatial memory to locate salient objects, as is the case when driving. Recent evidence suggests that the increased gaze movements including larger scanpath length, more gaze shifts, larger saccadic amplitudes, and more repetitive fixations allows persons with hemianopia to cope with the increased demands of more complex tasks and perform at similar levels to that of controls, ${ }^{13}$ which supports our finding that the bias of head movements into the blind rather than the seeing field was most apparent for those drivers rated as safe to drive.
The rating of the stability of lane positioning derived from the video footage was significantly worse for those drivers with field defects categorized as unsafe to drive compared with those rated as safe, which is in accord with the subjective ratings of the masked backseat rater and CDRS in this study as reported previously. ${ }^{5,18}$ Although previous studies have not determined whether lane positioning characteristics vary between safe and unsafe hemianopic drivers, they have reported greater numbers of lane crossing for those with hemianopia compared with controls in a simulator ${ }^{1}$ and in steering stability in an on-road assessment. ${ }^{2}$ We also demonstrated that those with hemianopic defects who were rated as unsafe to drive adopted a lane position that was toward their seeing field; that is, those with right hemianopia drove toward the left side of the lane and vice versa, which confirms the findings of a recent driving simulator study. ${ }^{16}$

The objective vehicle control measures from the instrumentation installed in the vehicle also demonstrated that participants with hemianopic or quadrantanopic defects drove significantly more slowly and had less jerky acceleration and less jerky cornering than did those with normal fields. This suggests that the hemianopic and quadrantanopic patients attempted to compensate for their visual field defects by driving more slowly, a strategy that has been reported in a number of other studies of real-world driving studies for persons with simulated $^{5,19}$ and true visual impairment ${ }^{2,20}$ and in simulator studies of hemianopic drivers. ${ }^{3}$ Although the unsafe hemianopic and quadrantanopic drivers drove more slowly than those who were rated as safe, they also exhibited twice as many sudden braking events than did the safe drivers. Thus the compensatory action of slowing was not always sufficient to avoid having to brake suddenly in response to events occurring within the driving environment.

The results of this study should be considered in the light of several limitations. First, the number of study participants was relatively small. Thus, though we observed distinct patterns of poorer performance among homonymous hemianopic and quadrantanopic drivers compared with those with normal fields and unsafe compared with safe drivers, many of these differences were not statistically significant. A second limitation was our use of a standardized but still subjective scheme for rating the safety of the drivers. Despite high inter-rater agreement of this scheme and its strong relationship with self-reported prospective crashes, ${ }^{21}$ its validity relative to more objective measures of driving safety (e.g., motor vehicle collisions) has yet to be established. Finally, the qualitative nature of the eye and head movement measures, which were derived from assessment of video footage of drivers rather than by using a formal eye and head tracker system, represents a potential limitation. However, it is important to note that the levels of inter-rater and intra-rater agreement were relatively high. The use of formal eye and head tracking systems under 
real-world driving conditions presents a range of technical problems in terms of changing illumination conditions, and calibration issues, as well as problems in using head-mounted systems when drivers are interacting in a normal traffic environment. In addition, the majority of eye trackers have problems in capturing high-quality images when participants are wearing spectacle lenses, because of additional reflections from the lenses themselves. Nevertheless, this study is the first to have attempted to characterize eye and head movement patterns of drivers with hemianopic or quadrantanopic field loss during actual on-road driving, and as such provides an important basis for future studies of eye and head movement patterns of persons with homonymous hemianopic or quadrantanopic defects during on-road driving performance.

In summary, as a group, persons with homonymous hemianopia and quadrantanopia made significantly more shoulder movements than did those with normal visual fields, and there was also a trend for more head movements in the hemianopes than in those with either quadrantanopia or normal visual fields. Those hemianopic and quadrantanopic drivers rated as safe to drive also exhibited different patterns of eye and head movements and driving characteristics compared with those rated as unsafe. They made more head movements into their blind than seeing field and were rated as having significantly more excursive eye movements and stable lane positioning, drove at higher speeds, and exhibited less sudden braking than those rated as unsafe. Future research needs to evaluate whether some of these characteristics could be trained in rehabilitation programs aimed at improving driving safety in this population. The potential for training the scanning patterns of persons with hemianopic field defects has been demonstrated in small-sample case series ${ }^{6,8-10}$ but has yet to be explored in terms of its ability to transfer road safety benefits to these patients.

\section{References}

1. Szlyk JP, Brigell M, Seiple W. Effects of age and hemianopic visual field loss on driving. Optom Vis Sci. 1993;70:1031-1037.

2. Tant MLM, Brouwer WH, Cornelissen FW, Kooijiman AC. Driving and visuospatial performance in people with hemianopia. Neuropsychol Rehab. 2002;12:419-437.

3. Bowers AR, Mandel AJ, Goldstein RB, Peli E. Driving with hemianopia, I: detection performance in a driving simulator. Invest $O p h$ thalmol Vis Sci. 2009;50:5137-5147.

4. Racette L, Casson EJ. The impact of visual field loss on driving performance: evidence from on-road driving assessments. Optom Vis Sci. 2005;82:668-674.

5. Wood JM, McGwin G Jr, Elgin J, et al. On-road driving performance by persons with hemianopia and quadrantanopia. Invest Ophthalmol Vis Sci. 2009;50:577-585.
6. Modi SC, Woo SY, Anderson EA, Strowmatt C, Perez AM. Low vision rehabilitation and driving training for hemianopic visual field loss: two case reports. International Congress Series. 2005; 1282:664-668.

7. Zihl J. Visual scanning behavior in patients with homonymous hemianopia. Neuropsychologia. 1995;33:287-303.

8. Kerkhoff $\mathrm{G}$. Neurovisual rehabilitation: recent developments and future directions. J Neurol Neurosurg Psychiatry. 2000;68:691706.

9. Spitzyna GA, Wise RJ, McDonald SA, et al. Optokinetic therapy improves text reading in patients with hemianopic alexia: a controlled trial. Neurology. 2007;68:1922-1930.

10. Roth T, Sokolov AN, Messias A, Roth P, Weller M, TrauzettelKlosinski S. Comparing explorative saccade and flicker training in hemianopia: a randomized controlled study. Neurology. 2009;72: 324-331.

11. Martin T, Riley ME, Kelly KN, Hayhoe M, Huxlin KR. Visuallyguided behavior of homonymous hemianopes in a naturalistic task. Vision Res. 2007;47:3434-3446.

12. Ishiai S, Furukawa T, Tsukagoshi H. Eye-fixation patterns in homonymous hemianopia and unilateral spatial neglect. Neuropsychologia. 1987;25:675-679.

13. Hardiess G, Papageorgiou E, Schiefer U, Mallot HA. Functional compensation of visual field deficits in hemianopic patients under the influence of different task demands. Vision Res. 50:11581172.

14. Pambakian AL, Wooding DS, Patel N, Morland AB, Kennard C, Mannan SK. Scanning the visual world: a study of patients with homonymous hemianopia. J Neurol Neurosurg Psychiatry. 2000; 69:751-759.

15. Riley ME, Kelly KN, Martin T, Hayhoe M, Huxlin KR. Homonymous hemianopia alters distribution of visual fixations in 3-dimensional visual environments. J Vision. 2007;7:289.

16. Bowers AR, Mandel AJ, Goldstein RB, Peli E. Driving with hemianopia, II: lane position and steering in a driving simulator. Invest Opbthalmol Vis Sci. 2010;51:6605-6613.

17. Halligan PW, Marshall JC, Wade DT. Visuospatial neglect: underlying factors and test sensitivity. Lancet. 1989;2:908-911.

18. Elgin JMG, Wood JM, Vaphiades MS, et al. Evaluation of on-road driving in people with hemianopia and quadrantanopia $A m J$ Occup Ther. 2010;64:1-11.

19. Higgins KE, Wood J, Tait A. Vision and driving: selective effect of optical blur on different driving tasks. Hum Factors. 1998;40:224232.

20. Wood JM. Age and visual impairment decrease driving performance as measured on a closed-road circuit. Hum Factors. 2002; 44:482- 494 .

21. Wood JM, Anstey KJ, Lacherez PF, Kerr GK, Mallon K, Lord SR. The on-road difficulties of older drivers and their relationship with self-reported motor vehicle crashes. J Am Geriatr Soc. 2009;57: 2062-2069. 